\title{
Utilización de otros láseres en Odontología: Argón, Nd:YAP y Ho:YAG

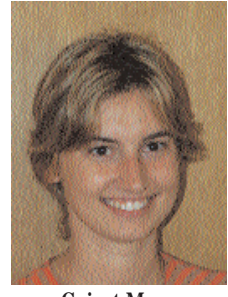 \\ Guinot-Moya, \\ Rosa
}

\section{Use of other lasers in dentistry: Argon, Nd:YAP and Ho:YAG}

\author{
Guinot-Moya, Rosa* \\ España-Tost, \\ Antonio Jesús** \\ Berini-Aytés, Leonardo***

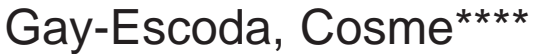

\begin{abstract}
* Odontólogo. Residente del Máster de Cirugía e Implantología Bucal. Facultad de Odontología de la Universidad de Barcelona.

** Médico Estomatólogo. Profesor Asociado de Cirugía Bucal y Profesor del Máster de Cirugía Bucal e Implantología Bucofacial. Facultad de Odontología de la Universidad de Barcelona.

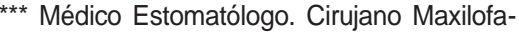
cial. Profesor Titular de Patología Quirúrgica Bucal y Maxilofacial. Profesor del Máster de Cirugía Bucal e Implantología Bucofacial. Facultad de Odontología de la Universidad de Barcelona.

**** Médico Estomatólogo. Cirujano Maxilofacial. Catedrático de Patología Quirúrgica Bucal y Maxilofacial. Director del Máster de Cirugía Bucal e Implantología Bucofacial. Facultad de Odontología de la Universidad de Barcelona. Cirujano Maxilofacial del Centro Médico Teknon. Barcelona.
\end{abstract}

\section{Correspondencia}

Cosme Gay Escoda Centro Médico Teknon

C/ Vilana 12

08022 Barcelona

E-mail: cgay@ub.edu

http:// www.gayescoda.com
Resumen: Las ventajas que la incorporación del láser ha proporcionado a la Odontología abarcan todos los ámbitos. Así, los láseres de Argón, de Nd:YAP y de Ho:YAG, tienen aplicaciones muy concretas y de gran interés tanto en el campo de la terapéutica dental como en la especialidad de cirugía bucal. Las aplicaciones principales del láser de Argón se centran en la polimerización de los materiales de restauración, en la endodoncia y dentro del ámbito de la cirugía bucal, en el corte de tejidos blandos, principalmente para la exéresis de lesiones vasculares y pigmentadas. El láser de Nd:YAP se utiliza principalmente en el campo de la endodoncia y la periodoncia, y el láser de Ho:YAG se emplea en cirugía periapical y en la cirugía artroscópica de la articulación temporomandibular.

Palabras clave: Láser de Argón, Láser de Nd:YAP, Láser de Ho:YAG, Láser en Odontología.

Abstract: The advantages that the incorporation of lasers brought to dentistry affect all fields. Thus, the argon lasers, Nd:YAP and Ho:YAG, have very specific and very interesting applications not only in operative dentistry but also in oral surgery. The indications of the argon laser are mainly focused on the polymerisation of restorative materials, in endodontics and within the oral surgery field, in the incision of soft tissues, specially in the treatment of vascular and pigmented lesions. The Nd:YAP laser is mainly applied in endodontics and periodontics, and the Ho:YAG is useful in periapical surgery and temporomandibular joint arthroscopic surgery.

Key words: Argon laser, Nd:YAP laser, Ho:YAG laser, Laser in dentistry.

\begin{tabular}{ccc}
\hline Fecha recepción & Fecha última revisión & Fecha aceptación \\
$25-4-2000$ & $26-2-2001$ & $19-3-2001$ \\
\hline
\end{tabular}

BIBLID [1138-123X (2004)9:5; septiembre-octubre 477-612]

Guinot-Moya R, España-Tost AJ, Berini-Aytés L, Gay-Escoda C Utilización de otros láseres en Odontología: Argón, Nd:YAP y Ho:YAG. RCOE 2004;9(5):581586 


\section{Introducción}

La incorporación de la tecnología láser en la Odontología ha revolucionado las técnicas de terapéutica dental y de la cirugía bucal. El láser ofrece multitud de ventajas, destacando en-tre otras, cursos postoperatorios más favorables, reducción de la cantidad de anestésico local necesario para que el paciente no tenga dolor y permitir que la sutura convencional sea prácticamente innecesaria en las intervenciones quirúrgicas.

En este artículo pretendemos mostrar las aplicaciones de algunos láseres de uso poco frecuente en Odontología, como son los láseres de Argón, de $\mathrm{Nd}$ :YAP y de Ho:YAG, pero que tienen algunas aplicaciones muy concretas en el campo de la terapéutica dental y de la cirugía bucal.

\section{Característica técnicas}

\section{Láser de Argón}

El láser de Argón emplea un gas como medio activo. Según la mezcla de gases de su cavidad de resonancia existen dos tipos de láseres de Argón, con longitudes de onda de 488 y 514 nm, comprendidas en el espectro azul y verde respectivamente, y con diferentes aplicaciones en el campo de la Odontología. Es conducido por una fibra óptica flexible, alcanzando potencias entre 0,5 y $25 \mathrm{~W}^{1}$.

Cuando es utilizado a mínima potencia provoca una buena polimerización de los materiales de restauración, mientras que a 1,5 W es un láser quirúr-

Tabla 1: Características técnicas del láser de Nd:YAP. Lokki

CARACTERÍSTICAS TÉCNICAS

Longitud de onda

Frecuencia

Energía por pulso

Duración del pulso

Potencia máxima

Diámetro de la fibra óptica
PARÁMETROS

$1340 \mathrm{~nm}$

$5-30 \mathrm{~Hz}$

$100-310 \mathrm{~mJ}$

$150 \mu \mathrm{s}$

$5 \mathrm{~W}$

240 y $385 \mu \mathrm{m}$ gico apropiado para el corte de tejidos blandos con mínimo sangrado y escaso dolor postoperatorio.

Puede emitir tanto en continuo como en pulsado y es absorbido por los pigmentos como la melanina, la hemoglobina y los carotenos. Su ab-sorción se produce fundamentalmente en la sangre, siendo por ello un excelente medio para facilitar la coagulación de los vasos $^{2}$.

El modelo LaserMed Accure 3000TM (Laser Med, Salt Lake City, EE.UU.) se utiliza para el cementado de las brackets de ortodoncia.

\section{Láser de Nd:YAP}

Es un láser con aplicación odontológica con una longitud de onda de 1340 $\mathrm{nm}$, situado dentro del espectro infrarrojo. Su medio activo, sólido, es una perusquita de itrio y aluminio, contaminada con iones trivalentes de neodimio. El rayo láser se aplica de modo pulsado a través de una fibra óptica de 200 a 300 $\mu \mathrm{m}$ que lo hace apropiado para el tratamiento de conductos. Emplea un rayo guía de He-Ne.

La casa comercial que distribuye este láser es Lokki (Vienne, Francia) y sus características técnicas se muestran en la tabla 1. Las características técnicas de este láser para las aplica- ciones endodóncicas las mostramos en la tabla 2. La figura 1 muestra el láser Looki y su panel de control.

\section{Láser de Ho:YAG}

Su longitud de onda, situada dentro del espectro infrarrojo, es de $2100 \mathrm{~nm}$. Su medio activo, sólido, es un granate de itrio y aluminio contaminado con holmium. Emplea un rayo guía de $\mathrm{He}-\mathrm{Ne}$ que se transmite por medio de una fibra óptica de modo pulsado.

\section{Aplicaciones clínicas}

\section{Láser de Argón}

Terapéutica Dental

\section{Polimerización}

Existen multitud de publicaciones sobre el uso del láser de Argón para obtener la fotopolimerización de las resinas. Todas ellas coinciden en destacar la reducción del tiempo de trabajo frente a las lámparas convencionales halógenas de fotopolimerizar (el láser de Argón tan solo requiere 10 segundos frente a los 40-60 de las lámparas halógenas $^{1,3^{*}}$. Asimismo destacan que las propiedades físicas ob-tenidas son 


\begin{tabular}{|lc|}
\hline \multicolumn{2}{|c|}{$\begin{array}{l}\text { Tabla 2: Características técnicas del láser de Nd:YAP } \\
\text { para uso en endodoncia }\end{array}$} \\
\hline CARACTERÍSTICAS TÉCNICAS & PARÁMETROS \\
\hline Frecuencia & $5 \mathrm{~Hz}$ \\
\hline Energía por pulso & $120-200-260 \mathrm{~mJ}$ \\
\hline
\end{tabular}
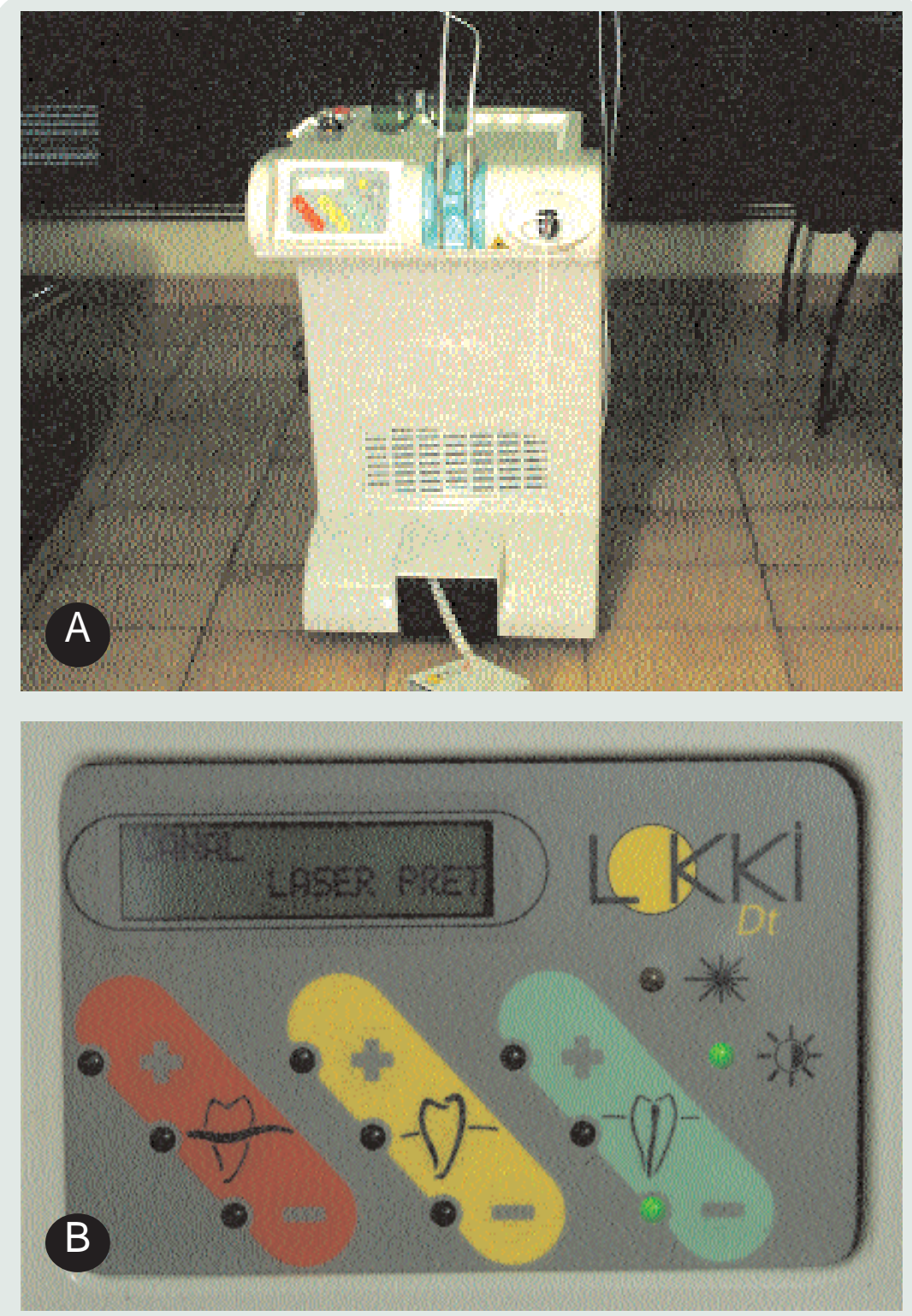

Figura 1. (A) Láser Lokki. (B) Tablero de mandos del láser Lokki. superiores en cuanto a fuerza, adhesión y adaptación marginal porque existe menor contracción tras la polimerización.

El láser de Argón produce menos irritación pulpar ya que deja menos cantidad de monómero libre, que es el causante de la irritación pulpar al quedar en contacto con los túbulos dentinarios ${ }^{1,3^{*}}$.

También se han publicado estudios sobre los efectos del láser de Argón sobre otros materiales fotopolimerizables como los ionómeros de vidrio, los adhesivos dentinarios, las bases cavitarias o los selladores de fosas y fisuras, con resultados satisfactorio ${ }^{3+4}$.

Una indicación muy interesante de este láser es su uso en las restauraciones de clase II, ya que el reducido tamaño de la fibra óptica permite un fácil acceso a la zona proximal de la caja de obturación ${ }^{1}$.

\section{Detección de caries.}

La aplicación del láser de Argón a mínima potencia sobre la superficie del diente, ilumina las zonas con ca-ries de color oscuro, mientras que las áreas descalcificadas aparecen con una fluorescencia rojo-anaranjada ${ }^{3^{*}}$.

3. Aumento de la resistencia a la caries

Se ha demostrado en algunos estudios tanto in vitro como in vivo, un aumento de la resistencia del esmalte irradiado con el láser de Ar-gón frente a los ácidos de la placa bacteriana. Una posible explicación de este hecho sería que la luz láser modifica la composición del esmalte disminuyendo su componente orgánico y de agua, aumentando su afinidad por el calcio, el hierro y los iones fosfato ${ }^{3^{*}}$. 


\begin{tabular}{|ll|}
\hline \multicolumn{2}{|c|}{ Tabla 3: Indicaciones del láser Lokki (Nd:YAP) } \\
\hline Operatoria Dental & - Tratamiento de caries \\
& - Acondicionamiento de esmalte y dentina \\
& - Pulpotomía \\
\hline Endodoncia & - Retratamiento endodóncico \\
& - Remoción de instrumentos fracturados \\
& - Remoción de postes \\
& - Hemostasia en endodoncia \\
\hline Periodoncia & - Tratamiento de las bolsas periodontales \\
& - Nuevos tratamientos conservadores \\
\hline Cirugía Bucal & - Hemostasia \\
& - Gingivectomía \\
& - Exéresis de épulis y otras lesiones de tejidos blandos \\
& - Operculectomía (exéresis capuchón mucoso) \\
& - Tratamiento de aftas \\
& - Blanqueamiento dental \\
& - Test de vitalidad pulpar
\end{tabular}

\section{Endodoncia}

La aplicación del láser de Argón en el interior del conducto radicular, en estudios realizados in vitro, han demostrado producir un sellado apical similar al obtenido con técnicas de condensación lateral y de gutapercha termoplastificada. Los resultados ob-tenidos en esta indicación por otros láseres como el de $\mathrm{CO}_{2}$ o el de $\mathrm{Nd}$ YYAG, han sido sustancialmente peores ${ }^{5,6}$.

\section{Odontopediatría}

Los efectos del láser de Argón sobre los tejidos pulpares no parecen ser nocivos al efectuar pulpotomías en dientes temporales. En un estudio de Wilkerson y cols se observó la respuesta clínica, radiográfica e histológica en 42 pulpotomías en dientes temporales, manteniéndose en todos los casos la vitalidad pulpar?

\section{Cirugía bucal}

Las indicaciones en esta especialidad se basan en su acción de corte de los tejidos blandos. El láser de Argón está indicado para la exéresis de fibromas, hiperplasias gingivales, para hacer vestibuloplastias, para la eliminación de excesos de tejidos blandos alrededor de las restauraciones dentarias y para el control del sangrado gingival alrededor de las preparaciones protésicas $^{1}$.

Otras aplicaciones interesantes son para la exéresis-biopsia de lesiones pigmentadas y vasculares de la cavidad bucal $^{8}$.

\section{Blanqueamiento dental}

El láser de Argón se ha empleado también con éxito en técnicas de blanqueamiento dental produciendo menor incremento de la temperatura pulpar que otros láseres ${ }^{9}$.

\section{Láser de Nd:YAP}

Las indicaciones principales de este tipo de láser se centran en el campo de la endodoncia, con el fin de obtener distintos beneficios y para efectuar diferentes técnicas:

\section{Efecto bactericida}

Se han efectuado algunos estudios para valorar la desinfección de los conductos radiculares contaminados con Streptococcus, comparando la clásica irrigación con hipoclorito de sodio, con la aplicación del láser de Nd:YAP10. Los resultados obtenidos indican que la aplicación de este láser a $1 \mathrm{~W}$ de potencia con una frecuencia de $30 \mathrm{~Hz}$ es tan eficaz como la aplicación de hipoclorito al 5,25\%, completada con la instrumentación manual, mientras que a frecuencias inferiores no se mostró tan efectivo.

\section{Preparación del conducto radicular}

La aplicación del láser de Nd:YAP de forma aislada no ha resultado eficaz para conseguir una correcta preparación biomecánica del conducto radicular, ya que no se obtiene una preparación de forma cónica y además aparecen gran cantidad de detritus ${ }^{11}$. Sin embargo ha resultado ser muy útil en el tratamiento y el retratamiento de los conductos radiculares cuando se ha completado la instrumentación manual con limas $\mathrm{k}^{12,13}$.

Eliminación de los materiales de restauración previamente al retratamiento endodóncico

El láser de Nd:YAP puede ser útil para retirar los materiales de obturación previa o simultánea al retratamiento endodóncico. Se ha demostrado en algún estudio que el láser de Nd:YAP es 
rápidamente absorbido por el material de restauración cuando la fibra se aplica en contacto directo, y que cuando se aplica a distancia debilita el material lo que facilita el posterior uso de los ultrasonidos $^{14}$

\section{Otras aplicaciones}

Existen otras indicaciones de este láser, que si bien están descritas por la casa comercial, en las áreas de periodoncia, cirugía bucal, terapéutica dental y prótesis, así como para conseguir el blanqueamiento dental, no cuentan con el respaldo bibliográfico suficiente que permita comparar la utilización de este láser frente a las técnicas convencionales. Estas aplicaciones se muestran en la tabla 3.

\section{Láser de Ho:YAG}

\section{Cirugía periapical}

Aunque se ha utilizado el láser de Ho:YAG para efectuar la sección apical (apicectomía) en cirugía periapical, exis- ten otros láseres más recomendables para esta indicación, como por ejemplo el láser de Er:YAG ${ }^{15}$ o el láser de Er,Cr:YSGG. Se ha observado en estudios realizados en dientes extraídos que la aplicación del láser de Ho:YAG sobre la raíz provoca algunos signos importantes de daño térmico, así como fisuras en la superficie radicular y huecos entre el conducto radicular y la gutapercha por contracción de esta última.

\section{Cirugía artroscópica}

Se ha utilizado ampliamente el láser de Ho:YAG en la cirugía artroscópica de la articulación temporomandibular, presentando grandes ventajas respecto a la instrumentación mecánica ${ }^{16^{\text {t*}}}$ : disminuye el tiempo operatorio, el acceso es fácil y cómodo por el reducido tamaño de la pieza de mano, produce un mínimo daño tisular, la precisión en la manipulación es muy buena, se reduce de forma importante la pérdida de sangre, disminuye el hematoma y el dolor postoperatorios y el tiempo de recupera- ción del pa-ciente es menor. Aún así, comparado con otros tipos de láseres, el de Er:YAG parece presentar menores efectos fototérmicos y fotomecánicos colaterales ${ }^{17}$.

\section{Terapéutica dental}

Se han publicado algunos estudios que evalúan la posible aplicación del láser de Ho:YAG en la preparación de cavidades, para hacer el grabado del esmalte dentario y para la eliminación de las caries. Al irradiar el esmalte y la dentina se consiguen perforaciones de aspecto homogéneo sin carbonización, y a baja potencia, una superficie fundida y recristalizada, que podría estar indicada para el tratamiento de la hipersensibilidad dentinaria ${ }^{18}$.

Si bien se ha propuesto su uso para diferentes tratamientos en terapéutica dental, la tendencia actual es utilizar otros láseres con menor efecto térmico, como por ejemplo el láser de Er:YAG y el láser de Er,Cr:YSGG.

\section{Bibliografía recomendada}

Para profundizar en la lectura de este tema, el/los autor/es considera/an interesantes los artículos que aparecen señalados del siguiente modo: *de interés ${ }^{*}$ de especial interés.

1. Powell, GL; Blankenau, RJ. Laser curing of dental materials. J Oral Laser Applications 2001;1:7-11.

2. España AJ, Velasco V, Gay Escoda C, Berini L, Arnabat J. Aplicaciones del Láser de $\mathrm{CO}_{2}$ en Odontología. Madrid: Ergon,1995.

3*. Verheyen, P. Photopolymerization with the argon laser. J Oral Laser Applications 2001;1:49-54. Este autor realizó la polimerización de diferentes materiales de restauración, empleando el láser de Argón y lo comparó con las lámparas halógenas convencionales. Obtuvo una disminución sustancial del tiempo de polimerización y una mejora en las propiedades mecánicas del material.

4. Powell, GL, Blankenau, RJ. Effects of argon laser curing on dentin shear bond strengths. J Clin Laser Med Surg 1996;3:111-7
5. Anic I, Matsumoto K. Comparison of the sealing ability of laser-softened, laterally condensed and low-temperature thermoplasticized gutta-percha. J Endod 1995;21:464-9.

6. Yamazaki R, Goya C, Tomita Y, Kimura Y, Matsumoto K. Study on apical leakage of the teeth after argon laser treatment and obturation. J Clin Laser Med Surg 1999;17:121-5.

7. Wilkerson MK, Hill SD, Arcoria CJ. Effects of the argon laser on primary tooth pulpotomies in swine. J Clin Laser Med Surg 1996; 14:37-42.

8. Gáspár, L. The use of four different lasers in oral soft tissue surgery. The fourth International Congress on Lasers in Dentistry 1994. Singapore.

9. Luk K, Tam L, Hubert M. Effect of light energy on peroxide tooth bleaching. J Am Dent Assoc 2004;135:194-201.

10. Blum JY, Michailesco P, Abadie MJ. An evaluation of the bactericidal effect of the Nd:YAP laser. J Endod 1997;23:583-5.

11. Blum JY, Abadie MJ. Study of the Nd:YAP laser. Effect on canal cleanliness. J Endod 1997;23:669-75.

12. Moshonov J, Peretz B, Brown T, Rotstein I. Cleaning of the root canal using $\mathrm{Nd}$ :YAP laser and its effect on the mineral content of the dentin. J Clin Laser Med Surg 2003;21:279-82.

13. Farge P, Nahas P, Bonin P. In vitro study of a Nd:YAP laser in endodontic retreatment. $\mathrm{J}$ Endod 1998;24:359-63. 
14. Blum JY, Peli JF, Abadie MJ. Effects of the Nd:YAP laser on coronal restorative materials: Implications for endodontic retreatment. J Endod 2000; 26: 588-92.

15. Komori T, Yokohama K, Matsumoto Y. Erbium:YAG and holmium:YAG laser root resection of extracted human teeth. J Clin Laser Med Surg 1997;15:9-13.

16**. Mazzonetto R, Spagnoli DB. Long-term evaluation of arthroscopic discectomy of the temporomandibular joint using the Hol-
mium:YAG laser. J Oral Maxillofac Surg 2001;59:1018-23.

Este estudio nos muestra la aplicación del láser de Ho:YAG en técnicas artroscópicas de la ATM en 30 pacientes que presentaban dolordisfunción temporomandibular, con un porcentaje de éxitos del 93,33\%, consiguiendo disminuir el dolor y aumentar el rango de movilidad articular.

17. Nuebler-Moritz M, Rüschoff J, Hering P. Comparative investigation involving the effects of
Erbium:YAG and Holmium:YAG laser radiation on temporomandibular joint tissue. The 4th International Congress on Lasers in Dentistry 1994. Singapore.

18. Eduardo C, Zezell D. Scanning electron microscopy analysis of enamel and dentine irradiated by Holmium Laser. The 4th International Congress on Lasers in Dentistry 1994. Singapore. 